\title{
XBP1 promotes tumor invasion and is associated with poor prognosis in oral squamous cell carcinoma
}

\author{
YANAN SUN ${ }^{1,2^{*}}$, FANGYAN JIANG ${ }^{1-3^{*}}$, YUEMEI PAN $^{1,2}$, XINMING CHEN $^{2}$, \\ JIRONG CHEN $^{1,2}$, YU WANG ${ }^{1,2}$, XUEQING ZHENG ${ }^{1,2}$ and JIALI ZHANG ${ }^{1,2}$
}

\author{
${ }^{1}$ Key Lab for Oral Biomedical Engineering of Ministry of Education, School and Hospital of Stomatology, \\ Wuhan University; ${ }^{2}$ Department of Oral Histopathology, School and Hospital of Stomatology, Wuhan University, \\ Wuhan, Hubei 430079; ${ }^{3}$ Department of Stomatology, Liuzhou General Hospital, Liuzhou, Guangxi 545006, P.R. China
}

Received September 15, 2017; Accepted May 31, 2018

DOI: $10.3892 / o r .2018 .6498$

\begin{abstract}
X-box-binding protein 1 (XBP1) contributes to various types of cancer including breast, bladder cancer and esophageal squamous cell carcinoma. The aim of the study was to examine the metastatic role of XBP1 in oral squamous cell carcinoma (OSCC), and identify possible downstream molecules. Immunohistochemical staining was conducted on tissue microarrays comprising 96 OSCC cases to determine the expression level of XBP1 and analyze its association with metastasis, clinicopathological characteristics and survival prognosis. Compared with the adjacent normal tissues of OSCC, the expression of XBP1 was significantly increased in the tumor center and front area, and lymph nodes metastases $(\mathrm{P}<0.05)$. A relatively high $\mathrm{XBP} 1$ expression was associated with histological grades $(\mathrm{P}<0.05)$, advanced clinical stages $(\mathrm{P}<0.05)$, unfavorable 5-year survival $(\mathrm{P}=0.027)$. Suppressed $\mathrm{XBP} 1$ expression caused a significant reduction of cell invasion capability $(\mathrm{P}<0.05)$. AXL and the downstream molecules, such as PI3K, MMP1, MMP3, and UPA were significantly suppressed when XBP1 expression was inhibited in OSCC cells. Once XBP1 was activated by Thapsigargin, AXL expression was restored. Moreover, aberrant AXL expression was associated with XBP1 overexpression in OSCC tissues $(\mathrm{P}<0.05)$. In conclusion, $\mathrm{XBP} 1$ is a potential target that is relevant to suppressing cell invasion and is associated with patient prognosis in OSCC.
\end{abstract}

\section{Introduction}

Oral squamous cell carcinoma (OSCC) is the one of the most common cancer types (1). With a propensity to lymph

Correspondence to: Professor Jiali Zhang, Department of Oral Histopathology, School and Hospital of Stomatology, Wuhan University, 237 Luoyu Road, Wuhan, Hubei 430079, P.R. China

E-mail: jiali_zhang@whu.edu.cn

*Contributed equally

Key words: XBP1, invasion, oral squamous cell carcinoma, prognosis, AXL node metastasis, the 5-year survival rate of OSCC is merely $40-50 \%$ (2). However, advances in surgical techniques as well as novel chemoradiation approaches remain less optimistic on the treatment of advanced OSCC (3), due to lymph node metastasis (4). Therefore, basic researches on OSCC focusing on tumor metastases are required, aiming to identify specific biomarkers that may offer novel therapeutic directions and new insight (5).

X-box-binding protein 1 (XBP1) belongs to the basic region/leucine zipper protein family, which is involved in unfolded protein response (UPR) (6). Once cleaved by inositol-requiring enzymela (IRE1) by removing 26-bp intron, and XBP1 mRNA fragments from the functional nuclear transcriptional factor, XBP1-s (spliced XBP1) (7,8). Previous findings showed that XBP1 is induced in various types of cancer, and controls cell type- and tissue-specific transcriptional regulatory networks in different cancer types (9-12). In addition, XBP1 was identified as a survival factor in certain malignant neoplasms $(13,14)$. Mounting evidence supported a direct role of XBP1 in tumor invasion, while loss of XBP1 was shown to severely inhibit tumor metastasis in vitro and in vivo (15). XBP1 also plays a pivotal role in tumor invasion through the upregulation of MMP9 expression in esophageal squamous cell carcinoma and triggering tumor EMT via promoting snail in breast cancer cells $(16,17)$. To the best of our knowledge, the role of XBP1 in OSCC metastasis and prognosis has not been clearly elaborated. Therefore, exploring the functional role and possible downstream signaling of XBP1 in OSCC is crucial to elucidate the effect of XBP1 on cancer development and progression.

The AXL receptor tyrosine kinase (AXL) is a member of the TAM (TYRO3-AXL-MER) family of receptor tyrosine kinases (18). A number of studies are available to support AXL as a candidate in tumor metastasis $(18,19)$ and cancer progression $(20,21)$. It is reported that AXL regulates cancer invasion in breast carcinoma $(19,22)$ and hepatocellular carcinoma (23) via the PI3K/Akt signaling pathway, by activating matrix metalloproteinase (MMPs) (24). Through genome-wide profiling, a recent study demonstrated that the expression of AXL was decreased when XBP1 expression was inhibited in XBP1-deficient breast cancer cells (9). However, no further research was performed to confirm the relationship between 
Table I. Patient characteristics.

\begin{tabular}{lcc}
\hline Group & Cases (96) & Percentage \% \\
\hline Sex & & \\
Male & 57 & 59 \\
Female & 39 & 41 \\
Age & & \\
$<55$ & 47 & 49 \\
$\geq 55$ & 49 & 51 \\
Location & & \\
Tongue & 34 & 35 \\
Other & 62 & 65 \\
Lymph node metastases & & \\
With & 20 & 21 \\
Without & 76 & 79 \\
Pathological grades & & \\
I & 47 & 13 \\
II & 35 & 36 \\
III & 14 & 31 \\
Clinical stages & & \\
I & 31 & \\
II & 30 & \\
III & 23 & \\
IV & 12 & \\
\hline
\end{tabular}

AXL and XBP1. Therefore, the aim of the present study was to demonstrate the role of XBP1 in OSCC invasion and prognosis. Moreover, the relationship between XBP1 and AXL signaling was explored to gain better understanding of the possible downstream signaling regulated by XBP1 in OSCC.

\section{Materials and methods}

Cell culture. The Cal27 cell line (derived from a tongue SCC patient) and the 293.2 sus cell line were obtained from the American Type Culture Collection (ATCC, Manassas, VA, USA). The head and neck squamous cell carcinoma cell line, UM-SCC-23, was a gift of Dr Thomas E. Carey. The material transfer agreement of this cell line was from the University of Michigan in June, 2012 (25). UM-SCC-23 and Cal-27 cells were cultured in DMEM (Hyclone Laboratories, Logan, UT, USA), and 293.2 sus was cultured in RPMI-1640 medium (Hyclone Laboratories). Cells were cultured as previously described (26).

Patients and tissue microarrays. The 96 OSCC samples for microarray were obtained from patients that underwent primary surgery at the Stomatology Hospital of Wuhan University (Hubei, China) between January 2002 and February 2009. All the adjacent normal epithelium samples were obtained by local excision. Ethics approval from the Institutional Review Board of School and Hospital of Stomatology, Wuhan University was received for the examination of patient samples. Specimens were fixed with formalin (Promoter Biotechnology, Hubei, China) postoperatively, and embedded with paraffin before being converted into tissue microarray slides. The histological types and tumor grades were analyzed by two pathologists. There were 228 spots in all from 76 patients without lymph node metastasis: For each patient, three spots were selected, respectively, from normal adjacent epithelium, tumor center and tumor front, respectively. The 228 spots were then spread evenly on 3 slides. In 20 patients with lymph node metastasis, 4 spots were selected from normal adjacent epithelium, tumor center, tumor front, and lymph node metastases, respectively. Then, 80 spots in all were spread on one slide. The diameter of each spot was $2 \mathrm{~mm}$. Tissue microarrays were produced by Outdo Biotech (Shanghai, China) after the design. Of the 96 tumors, 47 (48.96\%) were in grade I, 35 (36.46\%) were in grade II, and 14 (14.58\%) were in grade III. The pathological grade classification of the tumors was according to the 7 th edition of the cancer staging manual published by the American Joint Committee on Cancer (27). Other information related to the samples is listed in Table I.

Immunohistochemical staining. Paraffin-embedded specimens were sliced into 4- $\mu \mathrm{m}$ sections. After deparaffinating, dehydrating and performing antigen retrieval with high pressure, to quench the endogenous peroxidase activity, the sections were incubated with $3 \%$ hydrogen superoxide for $20 \mathrm{~min}$ followed by blocking non-specific binding in $10 \%$ normal goat serum. Immunohistochemical staining was performed as follows: The sections were incubated overnight at $4^{\circ} \mathrm{C}$ with polyclonal rabbit anti-human XBP1 (1:300; cat. no. ab37152; Abcam, Cambridge, UK), then labeled with HRP secondary antibody (Universal streptavidin-peroxidase kit including endogenous peroxidase blocking agent, goat serum, biotin-labeled goat

Table II. Specific XBP1-shRNA target sequences

\begin{tabular}{ll}
\hline XBP1 & \multicolumn{2}{c}{ Sequences } \\
\hline -sh1 & A: 5'-CCGGGACCCAGTCATGTTCTTCAAACTCGAGTTTGAAGAACATGACTGGGTCTTTTTG-3' \\
& B: 5'-AATTCAAAAAGACCCAGTCATGTTCTTCAAACTCGAGTTTGAAGAACATGACTGGGTC-3' \\
-sh2 & A: 5'-CCGGGCGGTATTGACTCTTCAGATTCTCGAGAATCTGAAGAGTCAATACCGCTTTTTG-3' \\
& B: 5'-AATTCAAAAAGCGGTATTGACTCTTCAGATTCTCGAGAATCTGAAGAGTCAATACCGC-3' \\
-sh3 & A: 5'-CCGGGAACAGCAAGTGGTAGATTTACTCGAGTAAATCTACCACTTGCTGTTCTTTTTG-3' \\
& B: 5'-AATTCAAAAAGAACAGCAAGTGGTAGATTTACTCGAGTAAATCTACCACTTGCTGTTC-3'
\end{tabular}


anti-rabbit $\operatorname{IgG}$, and horseradish peroxidase-labeled streptavidin; cat. no. SP-9001; Zhongshan, Beijing, China) followed by DAB (Maxim, Fuzhou, Fujian, China) color reaction. The sections were then counterstained with haematoxylin.

Scoring system, hierarchical clustering and data visualization. The tissue microarray slices stained with XBP1 antibody were scanned using Aperio ScanScope CS scanner (Vista, CA, USA), and Aperio ImageScope (Version 11.2) was used for nuclear quantification. Four high-power fields of interests were selected in each spot for quantifying the average optical density. Each field was read and signed with four kinds of colors according to color gradation (red for strong positive, orange for moderate positive, yellow for weak positive, and blue for negative). The mean histoscore of nuclear and cytoplasm staining was calculated using the formula: [(intensity of strong positive) $\times 3+$ (intensity of moderate positive) $\times 2+$ (intensity of weak positive) x1)]/selected area/4 (28). The hierarchical analysis was achieved by the HemI 1.0 with average linkage based on Pearson's correlation coefficient (29).

Plasmids and stable transduction. For the gene knockdown study, the GV-248-XBP1-sh1, 2, 3 and GV-248-con lentiviral vectors were purchased from Shanghai GeneChem Co., Ltd. (Shangai, China). The GV-248-XBP1-shs plasmids were generated by inserting the oligonucleotide containing the specific shRNA target sequences into the GV-248 vector and verified by sequencing. Three shRNA sequence pairs are shown in Table II. A cocktail for each transduction was produced by mixing the constructed, envelope, and packaging plasmids with serum-free OPTI-MEM (Gibco; Thermo Fisher Scientific, Inc., Waltham, MA, USA), which was added to 293.2 sus cells dropwise. After $48 \mathrm{~h}$ of transduction, lentiviral particles were harvested and stored in $-80^{\circ} \mathrm{C}$. To stably infect the target cells, lentiviral particle solution with polybrene (Sigma-Aldrich; Merck KGaA, Darmstadt, Germany) was added to the 50-60\% confluent target cells. GFP fluorescence sorting was used to select transducted cells.

Reverse transcription-quantitative PCR (RT-qPCR). Total RNA was extracted from the OSCC cell lines using the HP total RNA isolation kit (Omega Biotek, Norcross, GA, USA). RNA was transcribed into cDNA using the Takara ${ }^{\circledR}$ RT reagent kit (Takara Biotechnology, Co., Ltd., Dalian, Japan), and gene expression was quantified by Roche FastStart Essential DNA Green Master (Roche, Basel, Switzerland). Primer sequences are shown in Table III. The cycling parameters used were $95^{\circ} \mathrm{C}$ for $15 \mathrm{~min}$; followed by 40 cycles of $95^{\circ} \mathrm{C}$ for $15 \mathrm{sec} ; 55^{\circ} \mathrm{C}$ for $30-40 \mathrm{sec}$ and $72^{\circ} \mathrm{C}$ for $30 \mathrm{sec}$. The mRNA expression was normalized to that of $\beta$-actin. The differential expression of mRNA between transduced and control cells was deduced from $2^{-\Delta \Delta \mathrm{Cq}}$, where $\Delta \Delta \mathrm{Cq}=\Delta \mathrm{Cq}$ transducted cells- $\Delta \mathrm{Cq}$ control cells.

Protein extraction and western blot analysis. The total proteins were isolated from the OSCC cell lines using RIPA buffer and western blot analysis was conducted as previously described (26). The membranes were then probed with anti-human XBP1 antibody (1:800; cat. no. ab37152; Abcam) and anti-human AXL antibody (1:500; cat. no. AF154; R\&D
Table III. List of primers for RT-qPCR.

Gene Primer sequence

$\beta$-actin

F: 5'-ACCAACTGGGACGACATGGAGAAA-3'

XBP1 F: 5'-CCTGGTTGCTGAAGAGGAGG-3' R: 5'-TAGCACAGCCTGGATAGCAACGTA-3'

R: 5'-CCATGGGGAGATGTTCTGGAG-3'

AXL F: 5'-TCAAGGTGGCTGTGAAGACGATGA-3'

R: 5'-AACCCTGGAAACAGACACCGATGA-3'

E-cadherin F: 5'-AAGTCAGTTCAGACTCCAGCC-3'

R: 5'- TGTAGCTCTCGGCGTCAAA-3'

N-cadherin F: 5'-TGAAACGGCGGGATAAAGAG-3'

R: 5'-GGCTCCACAGTATCTGGTTG-3'

Slug F: 5'-TGGTTGCTTCAAGGACACAT -3'

R: 5'-GTTGCAGTGAGGGCAAGAA-3'

Snail1 F: 5'-GCGAGCTGCAGGACTCTAAT-3'

R: 5'-GGACAGAGTCCCAGATGAGC-3'

Twist1 F: 5'-GCCGGAGACCTAGATGTCATT-3'

R: 5'-CACGCCCTGTTTCTTTGAAT-3'

MMP1 F: 5'-AAGGCCAGTATGCACAGCTT-3'

R: 5'-GGGCCACTATTTCTCCGCTT-3'

MMP2 F: 5'-TGATGGCATCGCTCAGATCC-3'

R: 5'-GGCCTCGTATACCGCATCAA-3'

MMP3 F: 5'-CACAGTTGGAGTTTGACCC-3'

R: 5'-TAAGCAGCAGCCCATTTG -3'

MMP9 F: 5'-TTTGAGTCCGGTGGACGATG-3'

R: 5'-GCTCCTCAAAGACCGAGTCC-3'

MMP12 F: 5'-TGCTGATGACATACGTGGCA-3'

R: 5'-AGGATTTGGCAAGCGTTGG-3'

LAMA1 F: 5'-TCTGGGGAGAGATGTTGTGT-3'

R: 5'-ACGTTTAAAAAGAGAGCCAGGG-3'

PPFIBP1 F: 5'-CAGGGAGGGAGGAGAGAAGG-3'

R: 5'-GCCTGCACTACACCATGTCA-3'

CXCL-1 F: 5'-TCACAGTGTGTGGTCAACAT-3'

R: 5'-AGCCCCTTTGTTCTAAGCCA-3'

CXCL-2 F: 5'-CACAGTGTGTGGTCAACATTTCT-3'

R: 5'-TGCTCTAACACAGAGGGAAACA-3'

MAX F: 5'-TGCTCTAACACAGAGGGAAACA-3'

R: 5'-CGGGATGCCTTCTCTCCTTG-3'

Stat3 F: 5'-ATCCTGGTGTCTCCACTGGT-3'

R: 5'-CCTGGGTCAGCTTCAGGATG-3'

JUN F: 5'-GTGCCGAAAAAGGAAGCTGG-3'

R: 5'-CTGCGTTAGCATGAGTTGGC-3'

PI3K F: 5'-TGCAGTTTTGGAAGCAGTCAC-3'

R: 5'-CTGGAATAAGAACTATTCCTGCTCA-3'

PPFIBP1 F: 5'-CAGGGAGGGAGGAGAGAAGG-3'

R: 5'-GCCTGCACTACACCATGTCA-3'

C-MYC F: 5'-TCCTCGGATTCTCTGCTCTC-3'

R: 5'-CTCTGACCTTTTGCCAGGAG-3'

IL-6 F: 5'-TCAATATTAGAGTCTCAACCCCCA-3'

R: 5'-GAGAAGGCAACTGGACCGAA-3' 
A

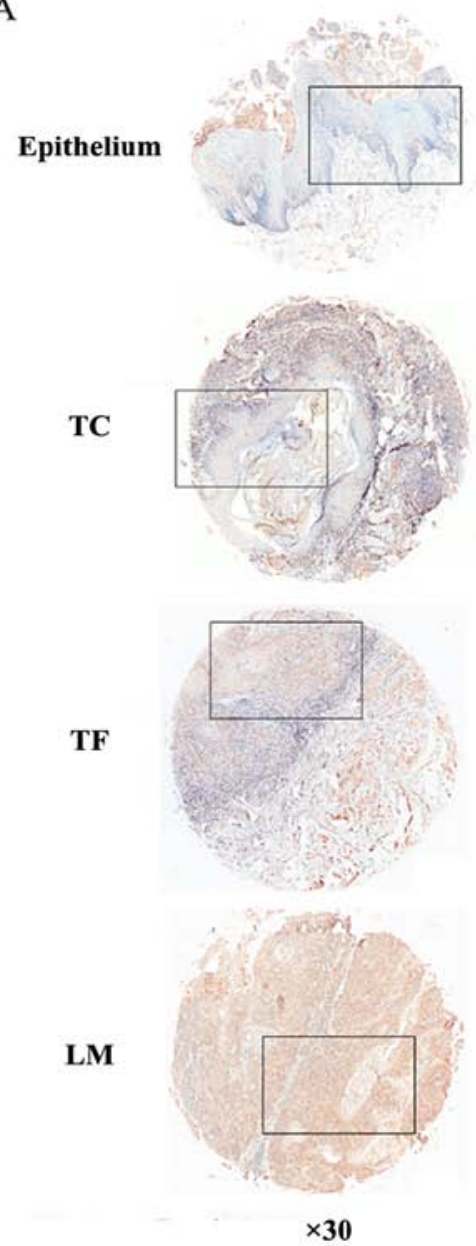

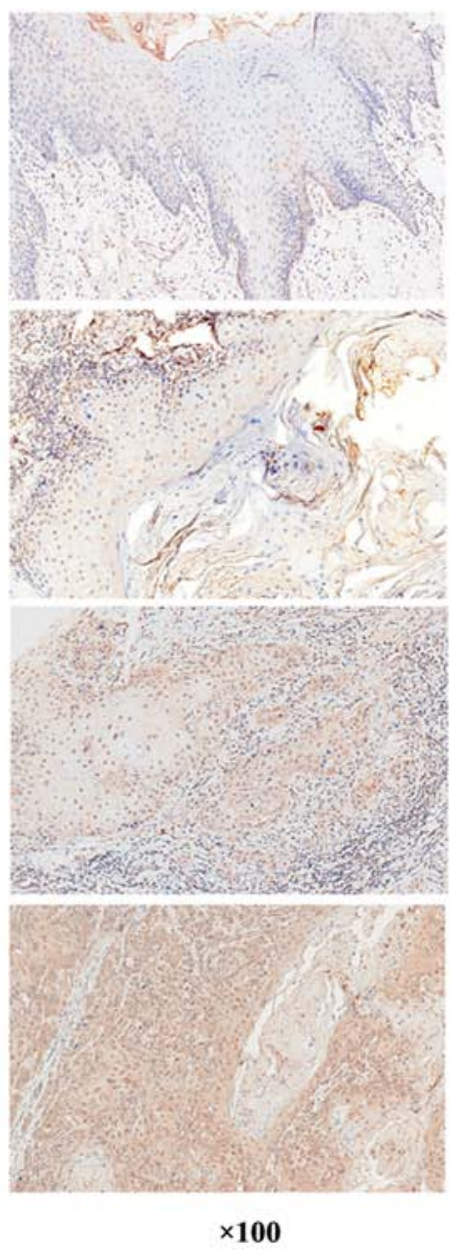

B

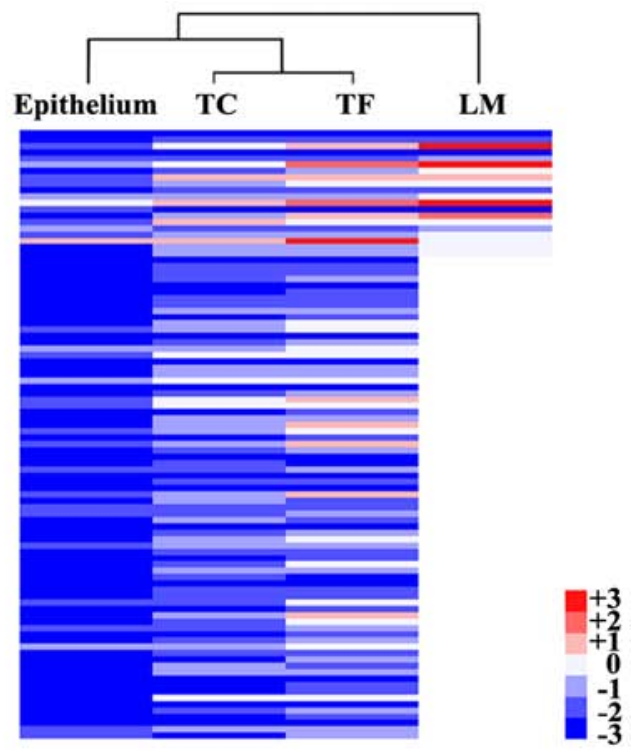

$\mathrm{C}$

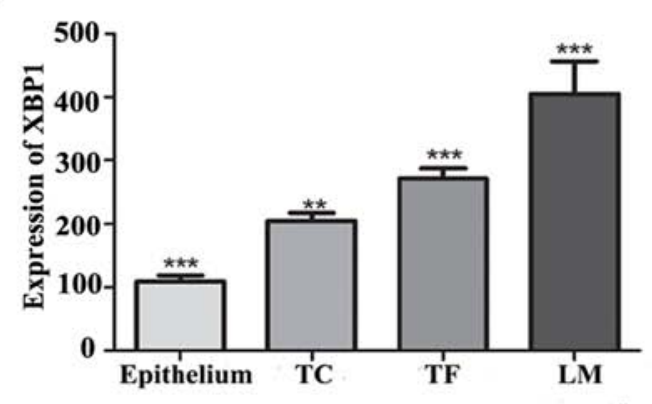

Figure 1. Expression of XBP1 in adjacent normal epithelium, tumor center, tumor front, and lymph node metastasis. (A) Immunohistochemical staining of XBP1 in representative cores of adjacent normal epithelium, tumor center (TC), tumor front (TF) and lymph nodes metastases (LM). Magnification, cores, $\mathrm{x} 30$; insets, $\mathrm{x} 100$. (B) Heat map visualization of XBP1 expression in epithelium, tumor center (TC), tumor front (TF) and lymph node metastasis (LM) in 96 OSCC cases. Hierarchical clustering was analyzed based on the results of IHC. Columns, cases; Rows, different areas of the tissue; color key indicates XBP1 expression value; blue, lowest; red, highest. (C) The histogram shows the expression of XBP1 presenting a significantly increasing trend in epithelium, tumor center (TC), tumor front (TF) and lymph node metastasis (LM). Significant differences were observed, respectively. ${ }^{* * *} \mathrm{P}<0.001 ;{ }^{* * *} \mathrm{P}<0.05$.

Systems, Minnneapolis, MN, USA) overnight at $4^{\circ} \mathrm{C}$. The membranes were also probed with anti- $\beta$-actin (Santa Cruz Biotechnology, Dallas, TX, USA) to ensure equal amounts of protein. Bound antibodies were detected using horseradish peroxidase-conjugated secondary antibody (Santa Cruz Biotechnology). Reactive protein was detected by ECL chemiluminescence system (Advanstar, Santa Monica, CA, USA). Western blot experiments were repeated in triplicate to confirm the results. The protein amounts were estimated through densitometry as the ratio detected protein $/ \beta$-actin.

Cell invasion assay. Matrigel-coated chamber (BD Biosciences Inc. San Jose, CA, USA) was prepared as previously described (26). Cells $\left(5 \times 10^{5}\right)$ were then seeded onto the Matrigel-coated chamber. Cancer cells were seeded using serum-free media to the upper chamber pairing with the lower chamber filled with $20 \%$ serum DMEM media. At $24 \mathrm{~h}$ after allowing cells to invade, cells on the lower side of the chamber were fixed in ethanol and stained with crystal violet (Guge Biotechnology, Wuhan, China). The total number of cells per high-power microscopic field on the lower side of Matrigel-coated chamber were counted and scored for invading cells. The mean number of cells in five high-power microscopic fields was calculated with standard deviations.

Thapsigargin stimulation and semi-quantitative RT-PCR. ER stress was induced in epithelial cells by exposure to thapsigargin. By the time of reaching near-confluence, the cells were exposed to thapsigargin (50 nM; Sigma-Aldrich; Merck KGaA) for $6 \mathrm{~h}$ (30). Dimethyl sulfoxide (DMSO; Merck KGaA) served as a solvent control for thapsigargin. To amplify the spliced and unspliced XBP1 mRNA, semi-quantitative RT-PCR was performed and XBP1 primers are shown in Table IV. PCR products were electrophoresed on $2.5 \%$ agarose gel and $\beta$-actin was used as a loading control. The size difference between the spliced and the unspliced XBP1 was 26 nucleotides.

Statistical analysis. Data analyses were performed using GraphPad Prism 5.0. One-way ANOVA followed by the Tukey's multiple comparison tests were performed to analyze 
Table IV. List of primers for semi-quantitative RT-PCR.

\begin{tabular}{ll}
\hline Gene & \multicolumn{1}{c}{ Primer sequence } \\
\hline$\beta$-actin & F: 5'-CCACCATGTACCCTGGCATT-3' \\
& R: 5'-CGCATCTCATATTTGGAATGACT-3' \\
XBP1 & F: 5'-CCTTGTAGTTGAGAACCAGG-3' \\
& R: 5'-GGGGCTTGGTATATATGTGG-3' \\
\hline
\end{tabular}

the differences in protein levels and invasion cell number among groups. The $\chi^{2}$ test was used to compare the dichotomous variables. The overall survival was analyzed by the Kaplan-Meier method and log-rank test. Data were presented as the mean \pm SEM. $\mathrm{P}<0.05$ was considered statistically significant for all tests.

\section{Results}

$X B P 1$ is increased in primary OSCC and lymph node metastasis. XBP1 expression was determined in tissue microarray using immunohistochemical staining. XBP1 protein mainly located in nuclei and/or cytoplasm presented as yellow or brown (Fig. 1A). The weak nuclei staining of XBP1 was observed in the adjacent normal epithelial (109.2 \pm 9.91 , mean \pm SEM). More intensive XBP1 expression was detected in tumor center (TC: $205.1 \pm 12.70$, mean \pm SEM) and tumor front (TF: 271.3 \pm 15.9 , mean \pm SEM) (Fig. 1C). Statistical analysis revealed that the relative expression level of XBP1 in the tumor center and tumor front was significantly higher than that in adjacent normal tissue $(\mathrm{P}<0.001)$. Moreover, compared with the tumor center, the tumor front exhibited a stronger XBP1 expression $(\mathrm{P}<0.05)$. In the samples with lymph node metastasis, the expression level of XBP1 showed a gradual increasing tendency from adjacent normal tissue, tumor center, and tumor front, to lymph node metastasis. The heatmap was performed to reveal the difference of XBP1 expression between normal adjacent epithelium, tumor center, tumor front, and lymph node metastasis (Fig. 1B). Statistically, the mean staining score of XBP1 in lymph node metastasis $(404.9 \pm 52.09$, mean \pm SEM) was 2.80 -fold of normal adjacent epithelial, 2.50-fold of tumor center and 1.83-fold of tumor front $(\mathrm{P}<0.001$, Fig. 1C).

High XBPl expression is associated with poor clinicopathological characteristics in OSCC patients. To determine the association between XBP1 protein expression level and the clinicopathological characteristics of OSCC patients, we analyzed the correlation between XBP1 expression and histological grades, clinical stages, sex and age. A high nuclei XBP1 expression level was detected in histological grade II $(187.5 \pm 15.03$, mean \pm SEM $)$ and III $(260.0 \pm 42.67$, mean \pm SEM), compared with histological grade I $(137.2 \pm 13.84$, mean \pm SEM). The expression level of XBP1 in grade III was 1.9-fold higher than that in grade I $(\mathrm{P}<0.001$, Fig. 2A). Additionally, the statistical data demonstrated that XBP1 expression was significantly correlated with advanced clinical stages $(\mathrm{P}<0.01$, Fig. 2B). Significantly stronger XBP1 staining was observed in stage III $(258.6 \pm 31.78$, mean \pm SEM $)$
$\mathbf{A}$

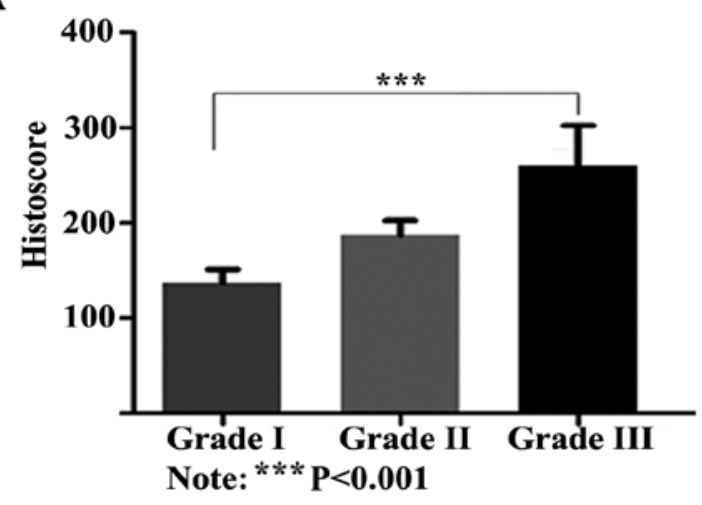

B

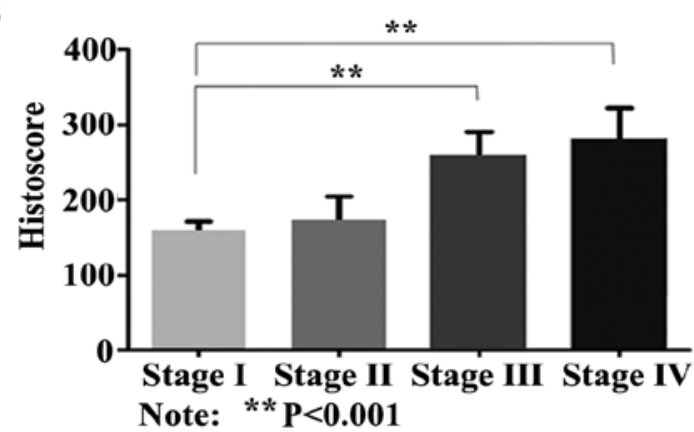

C

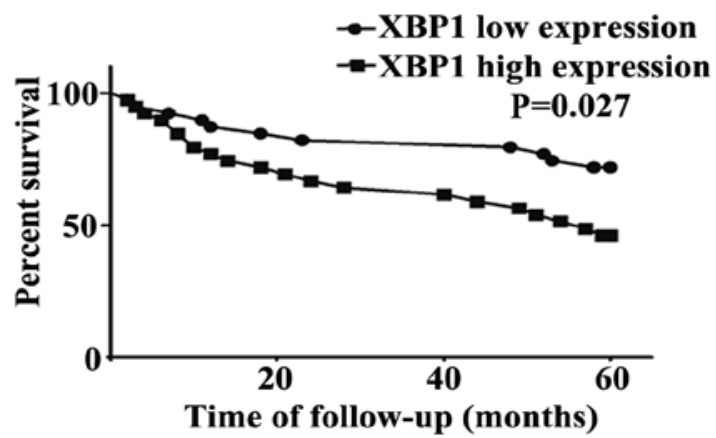

Figure 2. Quantitative analysis of XBP1 expression in tumor front area correlated with the higher pathological grades, advanced clinical stages, and poorer overall survival. (A) XBP1 expression level in tumor front was correlated with OSCC pathological differentiation grade $\left({ }^{* * *} \mathrm{P}<0.001\right)$. The expression level of XBP1 in grade III was significantly higher than that in grade I. (B) Stronger XBP1expression was observed in clinical stage III and IV than that in stage $\mathrm{I}\left({ }^{* *} \mathrm{P}<0.05\right)$. (C) Kaplan-Meier curve was generated to demonstrated a significant association between a relatively higher expression of XBP1 in tumor front and the poorer 5-year survival. The log-rank test was used to test for significant difference between the groups with $\mathrm{P}<0.05$.

and stage IV $(280.4 \pm 42.30$, mean \pm SEM $)$ than that in stage I $(159.6 \pm 11.91$, mean \pm SEM). However, no significant difference was detected between XBP1 expression and sex as or age.

High XBP1 expression predicts poor 5-year survival. To elicit the correlation between XBP1 expression and prognosis, the Kaplan-Meier survival analysis for XBP1 expression was performed. In total, 78 OSCC patients were followed up until death or more than 5 years (range, 60-111 months), and 18 patients were lost. During the follow-up period, 32 patients $(41.0 \%)$ succumbed to the disease within 60 months, 1 patient (1.3\%) succumbed to the disease more than 60 months 
$\mathbf{A}$

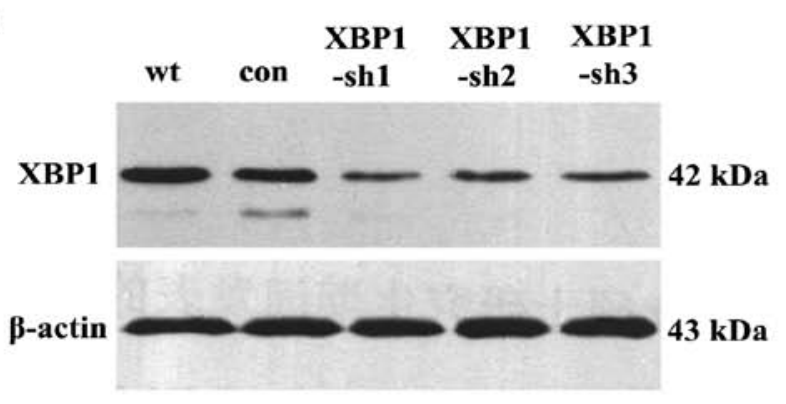

B

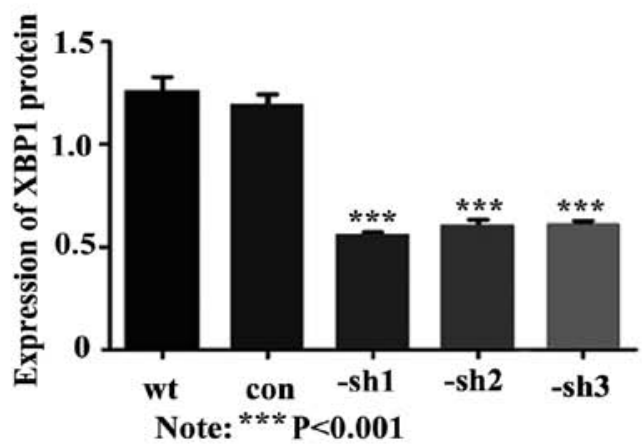

C
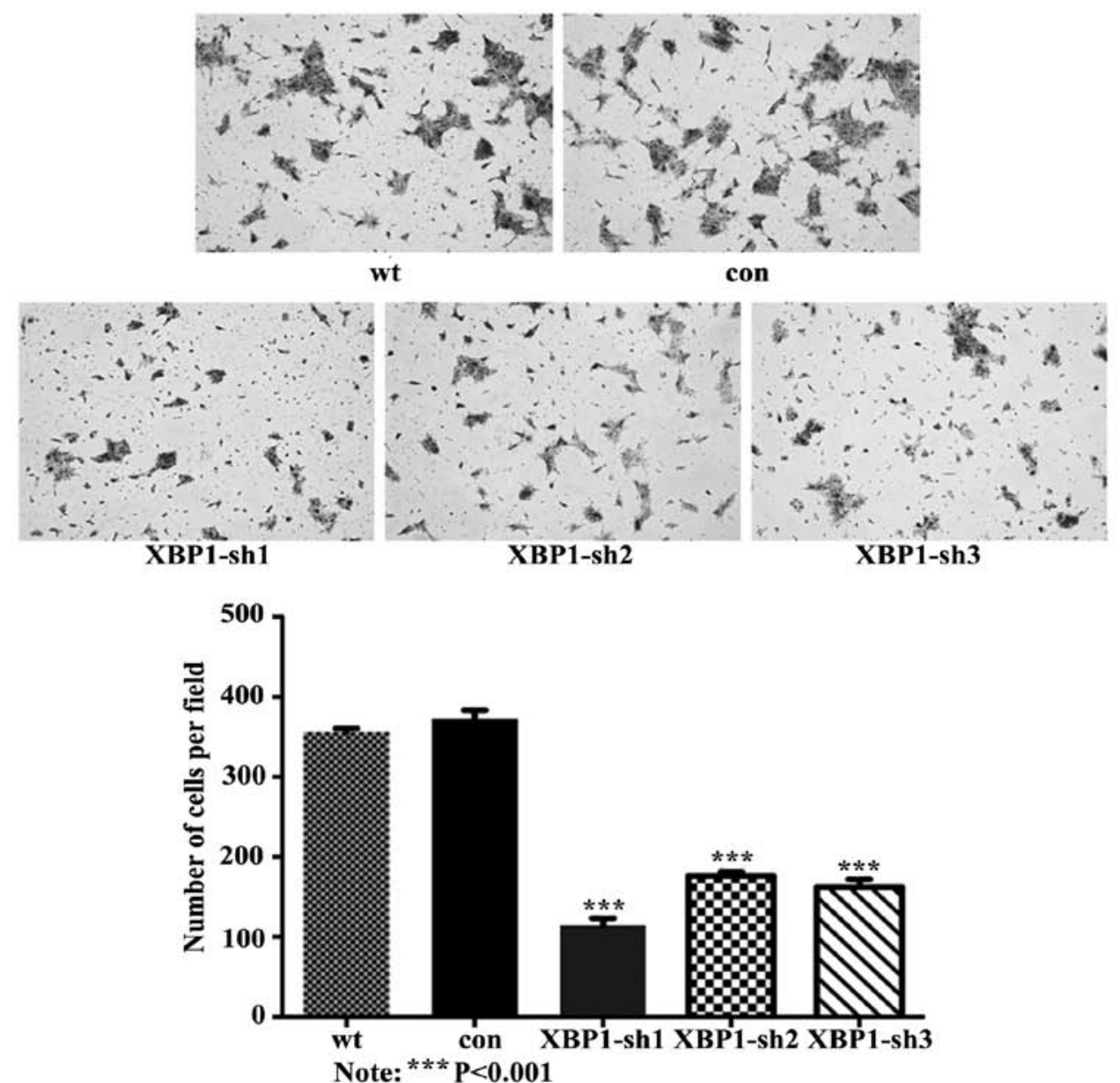

Figure 3. Attenuated XBP1 expression suppressed tumor cell invasion in vitro. (A) XBP1 protein expression was attenuated in UM-SCC-23 cells by stably knocking down XBP1 gene using three constructed lentiviral vectors GV-248-XBP1-sh1, 2, 3. (B) Expression of XBP1 in sh-1, 2 and 3 was decreased to 53, 70 and $75 \%$ of control, respectively $\left({ }^{* * *} \mathrm{P}<0.001\right)$. (C) The cell invasion capability was significantly reduced in XBP1-shs cells compared with control and wild-type cells $\left({ }^{* * *} \mathrm{P}<0.001\right)$. UM-SCC-23 cell invasion decreased approximately 3.25-, 2.11- and 2.29-fold when XBP1 expression was reduced using three different interference sequences. Original magnification, $\mathrm{x} 100$.

(77 months), and 45 patients $(57.7 \%)$ were alive. Based on the result of immunohistochemical staining, the samples with the tumor front staining score $\geq 222.65$ (median) were classified as high XBP1 expression, otherwise as low expression. The Kaplan-Meier survival analysis and the log-rank test showed that patients with a high level of XBP1 significantly were associated with unfavorable 5-year survival $(\mathrm{P}=0.027$, Fig. 2C).

Inhibition of XBP1 suppresses cell invasion in vitro. To further elucidate the function of XBP1 in the metastatic process of
OSCC, XBP1 were stably knocked down in SCC cells using three constructed lentiviral vectors GV-248-XBP1-sh1, 2, 3. The XBP1 protein expression was decreased significantly in XBP1-shs-transducted cells compared with the control $(\mathrm{P}<0.001$, Fig. 3A and B). The suppressed XBP1 expression caused a significant reduction of cell invasion capability (Fig. 3C). Statistically, the knockdown of XBP1 resulted in a 3.25-, 2.11- and 2.29-fold decrease of cell invasion, respectively, in UM-SCC-23-XBP1-sh1, -sh2 and -sh3, compared with the cells transduced with the control vector $(\mathrm{P}<0.001$, Fig. 3C). 
A

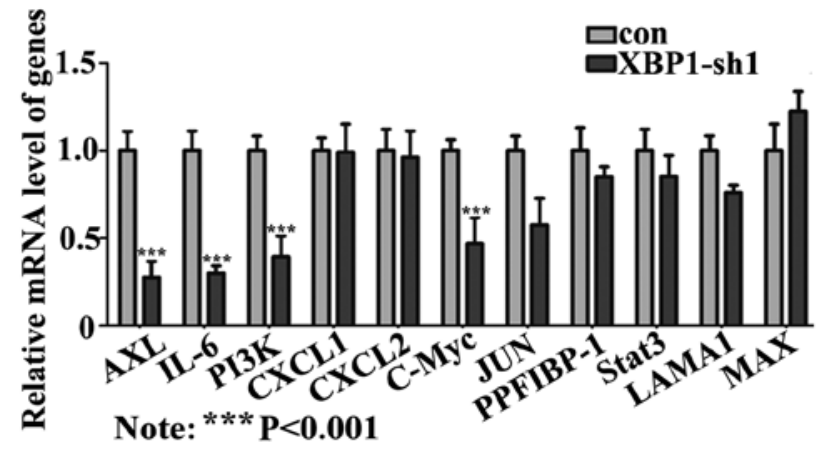

C

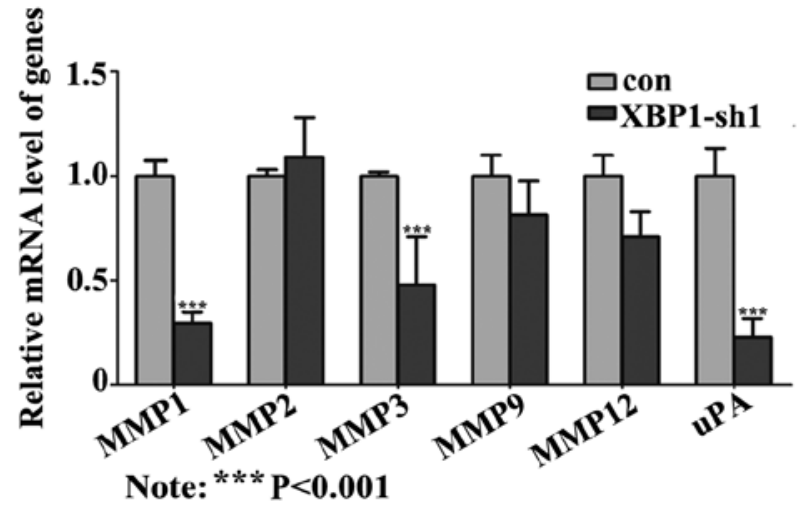

B

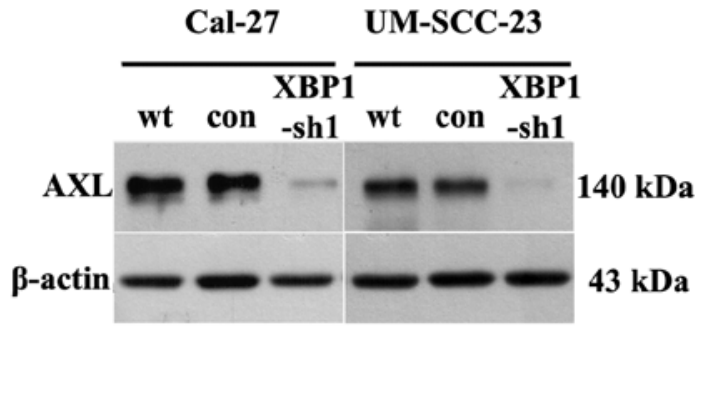

D

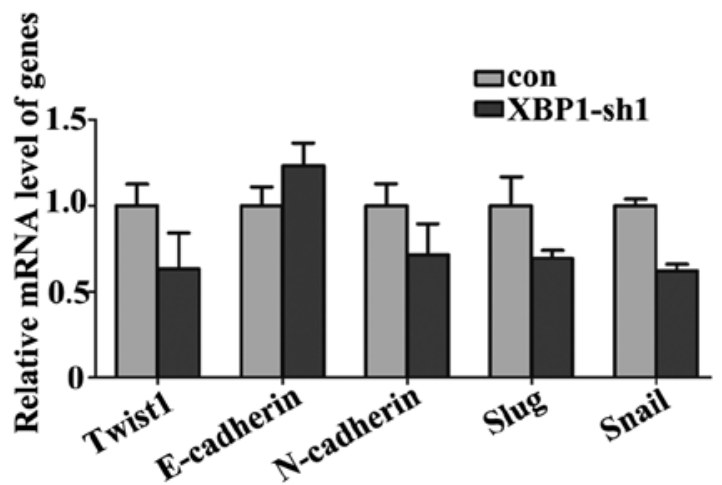

Figure 4. Suppression of XBP1 inhibits AXL signaling. (A) RT-qPCR results revealed the relative expression of 11 genes in Cal-27-XBP1-sh1 and Cal-27 control cell lines. The mRNA levels of AXL, PI3K, IL-6 and C-Myc were significantly reduced in XBP1-sh1 cells. (B) Expression of AXL was suppressed in UM-SCC-23-XBP1-sh1 and Cal-27-XBP1-sh1 cell lines, compared with the control cell lines in protein level. (C) The relative expression of MMP1, MMP3 and uPA were decreased significantly in Cal-27-XBP1-sh1 cell lines. (D) The relative mRNA expressions of EMT-related genes were detected in Cal-27-XBP1-sh1 and the control cell line, but no significant difference was found.

Suppression of XBP1 inhibits AXL signaling. To explore the possible downstream molecular factor of XBP1 involved in tumor metastasis, several invasion-related genes putatively implicated in XBP1 signaling according to the published data were investigated. The mRNA expression levels of 11 candidate genes were detected (Fig. 4A). Among these genes, the mRNA levels of AXL, PI3K, IL-6 and C-MYC were significantly reduced by more than 2-fold in XBP1-sh1 cells. Furthermore, the decreased expression of AXL in XBP1-shs cells was also confirmed in the protein level (Fig. 4B).

Recently, aberrant AXL was reported to be associated with tumor invasion by inducing MMPs or uPA or triggering EMT signaling (19,31). As a result, MMP1, 2, 3, 9, 12 and uPA, and the EMT-associated molecules, such as twist1, slug, snail, $\mathrm{E}$-cadherin and $\mathrm{N}$-cadherin were examined to evaluate the molecular contribution of XBP1 in OSCC invasion through AXL signaling. Significant reductions of MMP1, MMP3 and UPA were detected in XBP1-sh1 cells compared to the control cells (Fig. 4C). However, the mRNA expression of EMT markers showed no significant difference between cells (Cal-27) transduced with lentivirus encoding XBP1-sh1 and scramble vector (Fig. 4D).

$X B P 1$ activation rescued $A X L$ expression and promotes MMPs expression. Previous findings have demonstrated that XBP1 was regulated through a novel mechanism of mRNA splicing initiated by IRE1, an ER transmembrane kinase/endoribonuclease (32). To verify whether re-activation of XBP1 could rescue the expression of AXL, the ER stress inducer thapsigargin ( $\mathrm{Tg}$ ) was supplied to stimulate OSCC cells. After treating the cells with $50 \mathrm{nM} \mathrm{Tg}$ for $6 \mathrm{~h}$, the mRNA expression level of XBP1-s was significantly induced in XBP1-sh1 cells, as well as in control cells (Fig. 5A). Simultaneously, the protein levels of AXL were restored obviously when XBP1 was activated in XBP1-sh cells with Tg treatment (Fig. 5B). In addition, the mRNA expression levels of MMP1 and MMP3 were significantly increased in XBP1-sh1 cells treated with $50 \mathrm{nM}$ Tg for $6 \mathrm{~h}$, compared with cells treated with DMSO (Fig. 5C).

Aberrant AXL expression is correlated with XBPI overexpression in OSCC tissues. In order to evaluate the expression of AXL in OSCC tissues, the IHC staining was performed on tissue microarrays described before. Negative to weak cytoplasm staining of AXL was detected in the adjacent normal epithelium, while moderate to strong AXL staining was observed in the primary cancer cells and lymph node metastases. The expression levels of AXL in adjacent normal epithelium, primary cancer and lymph node metastasis showed an increasing tendency, which was consistent with XBP1 (Fig. 6A). To compare the relationship between the expression of AXL and XBP1 in OSCC, Spearman's correlation method was used to quantify the degree of linear association between two variables. The result showed that the levels of AXL were 
A

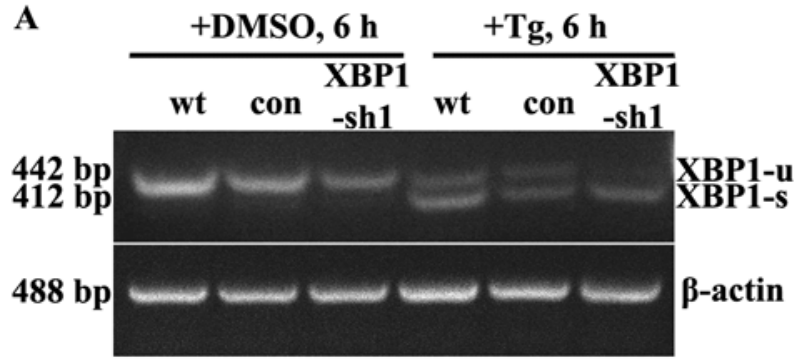

Cal-27

B

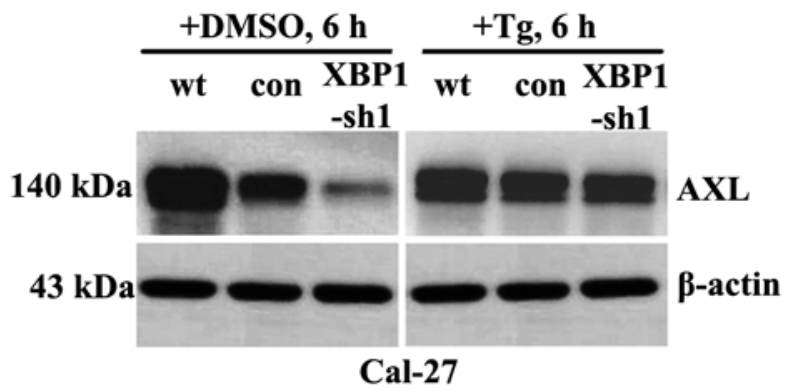

C

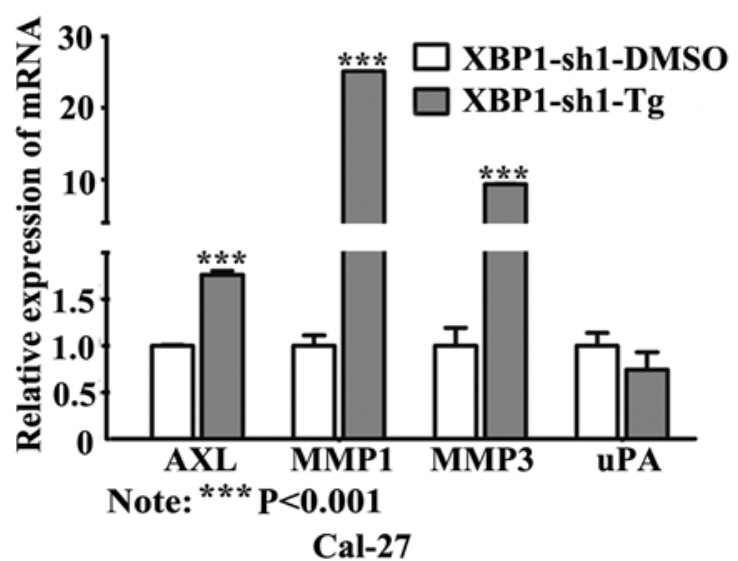

Figure 5. XBP1 activation rescued AXL expression. (A) Semi-quantitative PCR verified XBP1-s were induced by treating the Cal-27 cell line with $\mathrm{Tg}$ (50 nM, 6 h) compared with DMSO, $\beta$-actin was set as control. (B) AXL protein expression was restored in XBP1 knockdown cells by treating with Tg (50 nM, $6 \mathrm{~h})$. (C) RT-qPCR was performed to detect MMPs and uPA mRNA expression in Cal27-XBP1-sh1 cells treated with $50 \mathrm{nM}$ Tg for $6 \mathrm{~h}$. Cells treated with DMSO were set as the control. The relative expression of MMP1 and MMP3 was increased significantly in Tg-treated cell lines compared with the control group, but the expression of uPA showed a decreasing tendency.

positively correlated with XBP1 in primary cancer (Fig. 6B, $\mathrm{P}=0.0035$ ) and lymph node metastases (Fig. 6C, $\mathrm{P}=0.0211$ ), respectively.

\section{Discussion}

$X B P 1$, known as a candidate oncogenic gene, is overexpressed in certain cancers (13). The expression pattern and oncogenic function of XBP1 in OSCC is currently unclear. It was reported that unspliced forms of XBP1 (XBP1-u) mainly existed in the cytoplasm, and only the functional XBP1-s could translocate into the nucleus and trigger transcriptional programs by regulating a subset of genes involved in cancer progression (33). In the present study, XBP1 was detected overexpressed in both cytoplasm and nuclear in OSCC tumor cells compared with normal epithelial cells, with immunostaining being much stronger in nucleus. This result is in accordance with studies in esophageal SCC (16). Since the XBP1 antibody we used in this study was able to recognize both XBP1-s and XBP1-u, the location of XBP1 staining in the nucleus may indicate that the abundant XBP1 forms were spliced and activated in tumor cells and that its downstream transcriptional program may be initiated.

Previous findings have shown that ectopic overexpression of XBP1 resulted in metastasis in breast cancer $(6,9,17)$, esophageal SCC (16), and colorectal carcinoma (34). In the present study, we characterized the metastatic function of XBP1 in OSCC. Our findings demonstrated that XBP1 expression presented a rising tendency from normal epithelial to cancer metastasis. In lymph node metastasis, XBP1 showed the highest nuclear staining, followed by adjacent tumor front, tumor center, and adjacent normal epithelial. Moreover, transformed cells with XBP1 deficiency dramatically decreased OSCC cell invasion ability in vitro. These results suggest the effective function of XBP1 in promoting OSCC invasion and lymph node metastasis.

Recognition of $X B P 1$ target gene or downstream signaling is of great importance to understand the molecular mechanisms of XBP1-mediated tumorigenesis. It is demonstrated that XBP1 controls specific transcriptional signaling in specific cancer types. For example, XBP1 is reported to regulate the HIF1 $\alpha$ pathway in breast cancer (9), MMP9 in esophageal squamous cell carcinoma (16), $\beta$-catenin in bladder cancer (11), and the $\mathrm{PI} 3 \mathrm{~K} / \mathrm{mTOR}$ pathway in osteosarcoma (13), which are involved in cell proliferation, invasion and survival. The relationship between XBP1 and AXL was previously reported in breast cancer cells according to deep sequence data (9). However, to the best of our knowledge, no study has clarified the mechanism as to how XBP1 could directly control AXL expression. In our study, AXL was confirmed to be a novel downstream signaling of XBP1 in OSCC. We provide evidence that the expression of $A X L$ gene decreased at mRNA and protein levels when XBP1 expression was suppressed in OSCC cells. Conversely, activation of XBP1 by Tg immediately rescued the expression of AXL. Our result demonstrates AXL may be essentially involved in XBP1-mediated transcriptional events in OSCC. In addition, we demonstrated that the expression of AXL correlated with XBP1 positively in OSCC tumor center and lymph node metastasis. To better understand the downstream signal regulation of XBP1, further efforts are required to elucidate more detailed regulation mechanism between XBP1 and AXL.

AXL is detected as an essential biomarker in cancer progression associated with metastasis $(22,35,36)$, overall survival (18), and apoptosis (37). A crucial mechanism reported in endometrial cancer (35) is that AXL induces MMPs and uPA expression through the PI3K/AKT signaling pathway, thereby, promoting tumor metastasis. Several MMP genes have been identified to be regulated by AXL signaling which contribute to tumor invasion and metastasis. MMP2 and MMP9 are by far the most commonly reported tumor invasion-related genes associated with AXL pathway in the MMP family $(38,39)$. Besides these, MMP3 is activated by AXL in metastatic head and neck cancer (24). Moreover, both 
$\mathbf{A}$

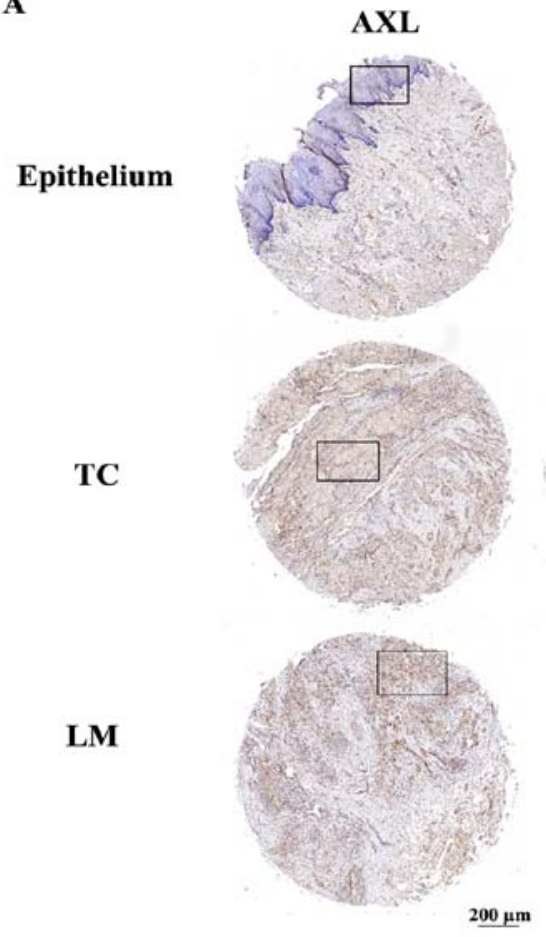

AXL
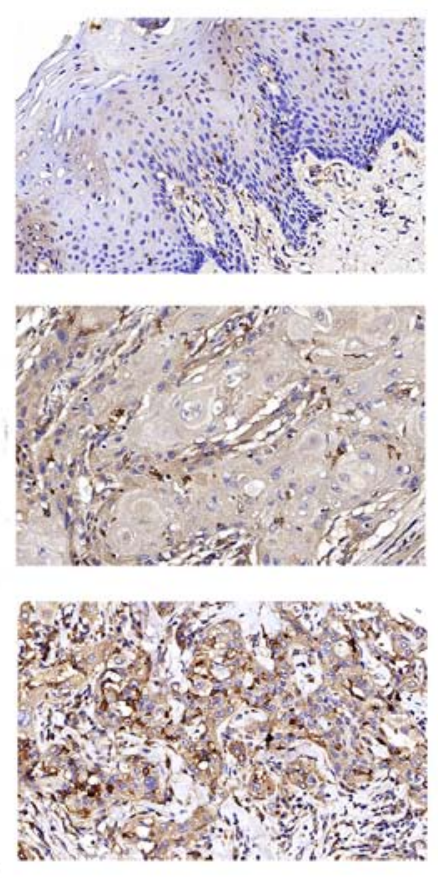
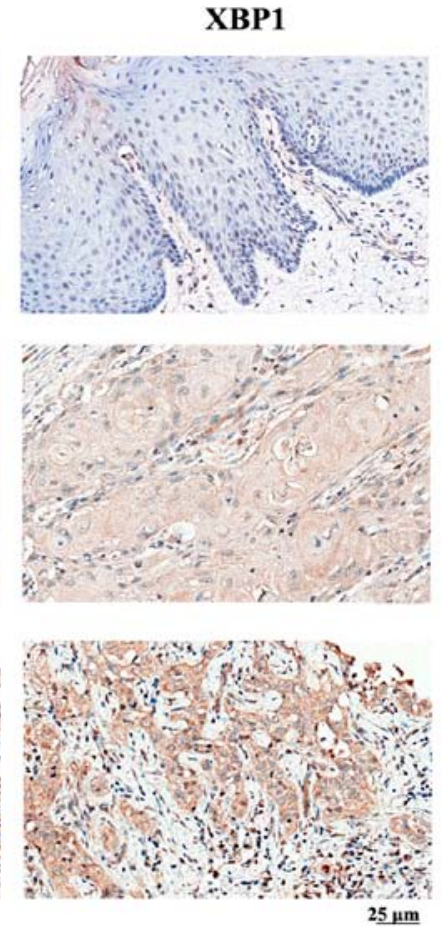

B

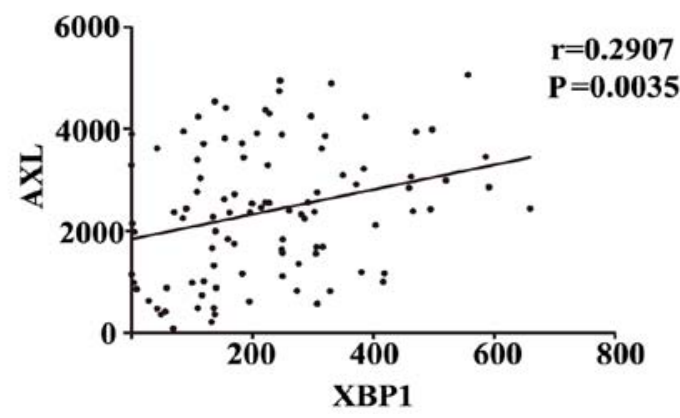

C

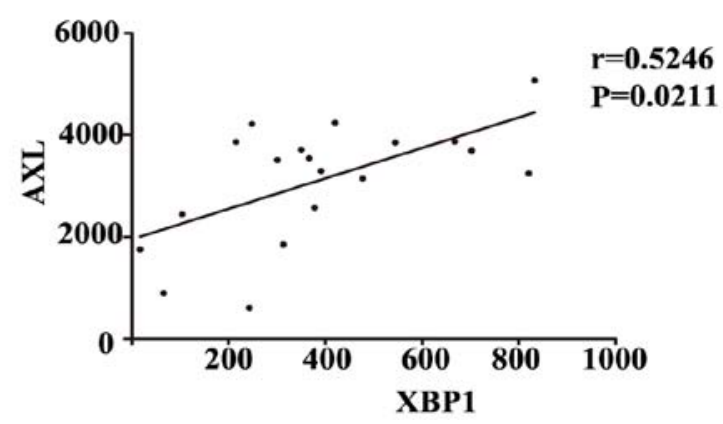

Figure 6. Aberrant AXL expression is correlated with XBP1 overexpression. (A) IHC staining showed that negative to weak cytoplasm staining of AXL was detected in the adjacent normal epithelium, while moderate to strong AXL staining was observed in cancer cells. The expression levels of AXL in adjacent normal epithelium, tumor center and lymph node metastasis showed an increasing tendency, which is in accordance with XBP1. (B) The level of AXL was positively correlated with XBP1 in tumor center $(\mathrm{P}=0.0035)$ and $(\mathrm{C})$ lymph node metastasis $(\mathrm{P}=0.0211)$.

MMP1 and MMP3 were regulated by AXL, resulting endometrial cancer metastasis (35). UPA is another crucial factor involved in extracellular matrix degradation through AXL signaling (35), which plays a major role in metastasis of cancer cells, by mediating directed extracellular proteolysis on the surface of migrating or invading cells. In the present study, we found that once XBP1 was suppressed in OSCC cell lines, several downstream molecules of AXL signaling, such as PI3K, MMP1, MMP3 and UPA, were significantly decreased, while MMP2 and MMP9 remained unchanged. Moreover, once XBP1 was re-activated in XBP1 knockdown cells, the expression levels of MMP1 and MMP3 were increased significantly. Thus, AXL as well as the downstream molecules PI3K, MMP1, MMP3 and uPA, may be closely associated with XBP1-initiated OSCC metastasis. On the other hand, although AXL has been reported to promote tumor metastasis via EMT in breast cancer (18), we were not able to demonstrate the effect of XBP1 on EMT-associated factors in XBP1 defection
OSCC cell lines. This indicates that XBP1 is not capable of significantly triggering tumor EMT phenotype in OSCC.

XBP1 was reported to be significantly correlated with clinical outcome in various tumors, such as human osteosarcoma (13), multiple myeloma (40), diffuse large B-cell lymphomas (41) and breast carcinoma (9). Therefore, the prognosis of patients with OSCC as well as the clinical pathology factors was taken into consideration in our study. After more than 5 years of follow-up, the findings of this study have shown that a relatively high XBP1 expression predicted unfavorable overall survival in OSCC patients. Moreover, XBP1 tends to show a higher expression in poorly differentiated tumor cells and advanced clinical stages. Thus, XBP1 is a potential survival factor in predicting OSCC patient outcome.

In summary, the above results demonstrate that $\mathrm{XBP} 1$ is a novel survival marker of OSCC, which was closely associated with tumor lymph node metastasis, advanced clinical stages, poor pathological differentiation and unfavorable prognosis. 
Aberrant XBP1 expression has a powerful effect on tumor invasion and apoptosis, and strongly modulates AXL signaling.

\section{Acknowledgements}

We would like to thank Shichun Xiong and Yuan Li of Wuhan University for their technical assistance and Professor T.E Carey of Michigan University for presenting UM-SCC-23 cell lines.

\section{Funding}

The present study was supported by the National Natural Science Foundation of China (grant no. 81572664).

\section{Availability of data and materials}

All data generated or analyzed during this study are included in this published article.

\section{Authors' contributions}

YS performed the construction of plasmid, verified the knockdown efficiency, conducted the invasion assay and examined the genes' expression downstream of XBP1; YS was a major contributor in acquisition of data and writing the manuscript; FJ participated in the construction of the TMA and performed IHC as well as the patients follow-up; YP undertook the task of cell culture; XC contributed to verify the histological types and tumor grades of tumor sample; JC shared the work of construction of TMA and patients follow-up; YW analyzed the data; XZ took part in the image processing; JZ made substantial contributions to the conception and design and to the acquisition of funding. JZ also contributed to the writing of the manuscript as well as the TMA construction. All authors read and approved the final manuscript. All authors read and approved the manuscript and agree to be accountable for all aspects of the research in ensuring that the accuracy or integrity of any part of the work are appropriately investigated and resolved.

\section{Ethics approval and consent to participate}

Ethics approval from the Institutional Review Board of School and Hospital of Stomatology, Wuhan University was received for the examination of patient samples.

\section{Patient consent for publication}

Not applicable.

\section{Competing interests}

The authors declare that they have no competing interests.

\section{References}

1. Fan S, Tang QL, Lin YJ, Chen WL, Li JS, Huang ZQ, Yang ZH, Wang YY, Zhang DM, Wang HJ, et al: A review of clinical and histological parameters associated with contralateral neck metastases in oral squamous cell carcinoma. Int J Oral Sci 3: 180-191, 2011.
2. Warnakulasuriya S: Global epidemiology of oral and oropharyngeal cancer. Oral Oncol 45: 309-316, 2009.

3. Russo D, Merolla F, Mascolo M, Ilardi G, Romano S, Varricchio S, Napolitano V, Celetti A, Postiglione L, Di Lorenzo PP, et al: FKBP51 Immunohistochemical expression: A new prognostic biomarker for OSCC? Int J Mol Sci 18: pii: E443, 2017.

4. Lingen MW, Kalmar JR, Karrison T and Speight PM: Critical evaluation of diagnostic aids for the detection of oral cancer. Oral Oncol 44: 10-22, 2008.

5. Arellano-Garcia ME, Hu S, Wang J, Henson B, Zhou H, Chia D and Wong DT: Multiplexed immunobead-based assay for detection of oral cancer protein biomarkers in saliva. Oral Dis 14: 705-712, 2008.

6. Andres SA and Wittliff JL: Relationships of ESR1 and XBP1 expression in human breast carcinoma and stromal cells isolated by laser capture microdissection compared to intact breast cancer tissue. Endocrine 40: 212-221, 2011.

7. Bright MD, Itzhak DN, Wardell CP, Morgan GJ and Davies FE: Cleavage of BLOC1S1 mRNA by IRE1 Is sequence specific, temporally separate from XBP1 splicing, and dispensable for cell viability under acute endoplasmic reticulum stress. Mol Cell Biol 35: 2186-2202, 2015.

8. Wang Y, Xing P, Cui W, Wang W, Cui Y, Ying G, Wang X and $\mathrm{Li}$ B: Acute endoplasmic reticulum stress-independent unconventional splicing of XBP1 mRNA in the nucleus of mammalian cells. Int J Mol Sci 16: 13302-13321, 2015.

9. Chen X, Iliopoulos D, Zhang Q, Tang Q, Greenblatt MB, Hatziapostolou M, Lim E, Tam WL, Ni M, Chen Y, et al: XBP1 promotes triple-negative breast cancer by controlling the HIF1alpha pathway. Nature 508: 103-107, 2014.

10. Zhu H, Abulimiti M, Liu H, Su XJ, Liu CH and Pei HP: RITA enhances irradiation-induced apoptosis in p53-defective cervical cancer cells via upregulation of IRE1alpha/XBP1 signaling. Oncol Rep 34: 1279-1288, 2015.

11. Chen W, Zhou J, Wu K, Huang J, Ding Y, Yun EJ, Wang B, Ding C, Hernandez E, Santoyo J, et al: Targeting XBP1-mediated $\beta$-catenin expression associated with bladder cancer with newly synthetic Oridonin analogues. Oncotarget 7: 56842-56854, 2016.

12. Bae J, Samur M, Munshi A, Hideshima T, Keskin D, Kimmelman A, Lee AH, Dranoff G, Anderson KC and Munshi NC: Heteroclitic XBP1 peptides evoke tumor-specific memory cytotoxic T lymphocytes against breast cancer, colon cancer, and pancreatic cancer cells. Oncoimmunology 3: e970914, 2014.

13. Yang J, Cheng D,Zhou S,Zhu B,Hu T and Yang Q: Overexpression of X-Box binding protein 1 (XBP1) correlates to poor prognosis and up-regulation of PI3K/mTOR in human osteosarcoma. Int J Mol Sci 16: 28635-28646, 2015.

14. Davies MP, Barraclough DL, Stewart C, Joyce KA, Eccles RM, Barraclough R, Rudland PS and Sibson DR: Expression and splicing of the unfolded protein response gene XBP-1 are significantly associated with clinical outcome of endocrine-treated breast cancer. Int J Cancer 123: 85-88, 2008.

15. Mhaidat NM, Alzoubi KH and Abushbak A: X-box binding protein 1 (XBP-1) enhances colorectal cancer cell invasion. J Chemotherapy 27: 167-173, 2015.

16. Xia T, Tong S, Fan K, Zhai W, Fang B, Wang SH and Wang JJ: XBP1 induces MMP-9 expression to promote proliferation and invasion in human esophageal squamous cell carcinoma. Am J Cancer Res 6: 2031-2040, 2016.

17. Li H, Chen X, Gao Y, Wu J, Zeng F and Song F: XBP1 induces snail expression to promote epithelial-to-mesenchymal transition and invasion of breast cancer cells. Cell Signal 27: 82-89, 2015.

18. Gjerdrum C, Tiron C, Høiby T, Stefansson I, Haugen H, Sandal T, Collett K, Li S, McCormack E, Gjertsen BT, et al: Axl is an essential epithelial-to-mesenchymal transition-induced regulator of breast cancer metastasis and patient survival. Proc Natl Acad Sci USA 107: 1124-1129, 2010.

19. Li Y, Jia L, Ren D, Liu C, Gong Y, Wang N, Zhang X and Zhao Y: Axl mediates tumor invasion and chemosensitivity through PI3K/Akt signaling pathway and is transcriptionally regulated by slug in breast carcinoma. IUBMB Life 66: 507-518, 2014.

20. Lee CH, Liu SY, Chou KC, Yeh CT, Shiah SG, Huang RY, Cheng JC, Yen CY and Shieh YS: Tumor-associated macrophages promote oral cancer progression through activation of the Axl signaling pathway. Ann Surg Oncol 21: 1031-1037, 2014. 
21. Rankin EB and Giaccia AJ: The receptor tyrosine kinase AXL in cancer progression. Cancers (Basel) 8: pii: E103, 2016.

22. Li Y, Jia L, Liu C, Gong Y, Ren D, Wang N, Zhang X and Zhao Y: Axl as a downstream effector of TGF- $\beta 1$ via PI3K/Akt-PAK1 signaling pathway promotes tumor invasion and chemoresistance in breast carcinoma. Tumor Biol 36: 1115-1127, 2015.

23. Xu J, Jia L, Ma H, Li Y, Ma Z and Zhao Y: Axl gene knockdown inhibits the metastasis properties of hepatocellular carcinoma via PI3K/Akt-PAK1 signal pathway. Tumor Biol 35: 3809-3817, 2014.

24. Xu H, Chen X, Huang J, Deng W, Zhong Q, Yue C, Wang P and Huang Z: Identification of GPR65, a novel regulator of matrix metalloproteinases using high through-put screening. Biochem Biophys Res Commun 436: 96-103, 2013.

25. Brenner JC, Graham MP, Kumar B, Saunders LM, Kupfer R, Lyons RH, Bradford CR and Carey TE: Genotyping of 73 UM-SCC head and neck squamous cell carcinoma cell lines. Head Neck 32: 417-426, 2010.

26. Zhang J, Wang Y, Chen X, Zhou Y, Jiang F, Chen J, Wang L and Zhang WF: MiR-34a suppresses amphiregulin and tumor metastatic potential of head and neck squamous cell carcinoma (HNSCC). Oncotarget 6: 7454-7469, 2015.

27. Edge SB and Compton CC: The American Joint Committee on Cancer: The 7th edition of the AJCC cancer staging manual and the future of TNM. Ann Surg Oncol 17: 1471-1474, 2010.

28. Ma SR, Wang WM, Huang CF, Zhang WF and Sun ZJ: Anterior gradient protein 2 expression in high grade head and neck squamous cell carcinoma correlated with cancer stem cell and epithelial mesenchymal transition. Oncotarget 6: 8807-8821, 2015.

29. Deng W, Wang Y, Liu Z, Cheng H and Xue Y: HemI: A toolkit for illustrating heatmaps. PLoS One 9: e111988, 2014.

30. van Schadewijk A, van't Wout EF, Stolk J and Hiemstra PS A quantitative method for detection of spliced X-box binding protein-1 (XBP1) mRNA as a measure of endoplasmic reticulum (ER) stress. Cell Stress Chaperones 17: 275-279, 2012.

31. Corno C, Gatti L, Lanzi C, Zaffaroni N, Colombo D and Perego P: Role of the receptor tyrosine kinase Axl and its targeting in cancer cells. Curr Med Chem 23: 1496-1512, 2016.

32. Castillo-Carranza DL, Zhang Y, Guerrero-Muñoz MJ, Kayed R, Rincon-Limas DE and Fernandez-Funez P: Differential activation of the ER stress factor XBP1 by oligomeric assemblies. Neurochem Res 37: 1707-1717, 2012.
33. Yoshida H, Oku M, Suzuki M and Mori K: pXBP1(U) encoded in XBP1 pre-mRNA negatively regulates unfolded protein response activator pXBP1(S) in mammalian ER stress response. J Cell Biol 172: 565-575, 2006

34. Jin C, Jin Z, Chen NZ, Lu M, Liu CB, Hu WL and Zheng CG: Activation of IRE1 $\alpha$-XBP1 pathway induces cell proliferation and invasion in colorectal carcinoma. Biochem Bioph Res Commun 470: 75-81, 2016.

35. Divine LM, Nguyen MR, Meller E, Desai RA, Arif B, Rankin EB, Bligard KH, Meyerson C, Hagemann IS, Massad M, et al: AXL modulates extracellular matrix protein expression and is essential for invasion and metastasis in endometrial cancer. Oncotarget 7: 77291-77305, 2016

36. Mudduluru G, Vajkoczy P and Allgayer H: Myeloid zinc finger 1 induces migration, invasion, and in vivo metastasis through Axl gene expression in solid cancer. Mol Cancer Res 8: $159-169,2010$

37. Cho CY, Huang JS, Shiah SG, Chung SY, Lay JD, Yang YY, Lai GM, Cheng AL, Chen LT and Chuang SE: Negative feedback regulation of AXL by miR-34a modulates apoptosis in lung cancer cells. RNA 22: 303-315, 2016.

38. Han J, Tian R, Yong B, Luo C, Tan P, Shen J and Peng T: Gas6/Axl mediates tumor cell apoptosis, migration and invasion and predicts the clinical outcome of osteosarcoma patients. Biochem Bioph Res Commun 435: 493-500, 2013.

39. Chiu KC, Lee CH, Liu SY, Yeh CT, Huang RY, Yuh DY, Cheng JC, Chou YT and Shieh YS: Protumoral effect of macrophage through Axl activation on mucoepidermoid carcinoma. J Oral Pathol Med 43: 538-544, 2014.

40. Chen L, Li Q, She T, Li H, Yue Y, Gao S, Yan T, Liu S, Ma J and Wang Y: IRE1 $\alpha-X B P 1$ signaling pathway, a potential therapeutic target in multiple myeloma. Leuk Res 49: 7-12, 2016.

41. Balague O, Mozos A, Martinez D, Hernandez L, Colomo L, MateJL, Teruya-Feldstein J,Lin O, Campo E, Lopez-Guillermo A, et al: Activation of the endoplasmic reticulum stress-associated transcription factor $\mathrm{x}$ box-binding protein-1 occurs in a subset of normal germinal-center B cells and in aggressive B-cell lymphomas with prognostic implications. Am J Pathol 174: 2337-2346, 2009. 